\title{
The feasibility of high-efficiency InAs/GaAs quantum dot intermediate band solar cells
}

\author{
A. Mellor ${ }^{a, *}$, A. Luque ${ }^{a}$, I. Tobías $^{a}$, A. Martí ${ }^{a}$ \\ a Instituto de Energía Solar, Universidad Politécnica de Madrid, Avda. Complutense 30, 28040 Madrid, Spain
}

\section{A R T I C L E I N F O}

\section{Article history:}

Received 17 March 2014

Received in revised form

3 July 2014

Accepted 4 July 2014

Available online 30 July 2014

Keywords:

Quantum dot

Intermediate band

High efficiency

Light trapping

\begin{abstract}
A B S T R A C T
In recent years, all the operating principles of intermediate band behaviour have been demonstrated in InAs/GaAs quantum dot (QD) solar cells. Having passed this hurdle, a new stage of research is underway, whose goal is to deliver QD solar cells with efficiencies above those of state-of-the-art single-gap devices. In this work, we demonstrate that this is possible, using the present InAs/GaAs QD system, if the QDs are made to be radiatively dominated, and if absorption enhancements are achieved by a combination of increasing the number of QDs and light trapping. A quantitative prediction is also made of the absorption enhancements required, suggesting that a 30 fold increase in the number of QDs and a light trapping enhancement of 10 are sufficient. Finally, insight is given into the relative merits of absorption enhancement via increasing QD numbers and via light trapping.
\end{abstract}

(c) 2014 Elsevier B.V. All rights reserved.

\section{Introduction}

The intermediate band solar cell (IBSC) is a high efficiency solar cell concept whose detailed balance efficiency limit has been calculated as 63\% [1] to be compared to the Shockley-Queisser limit of $41 \%$ [2] for conventional single-bandgap solar cells. IBSCs have been realized using semiconductor quantum dots (QDs) [3-9], highly mismatched alloys [10-12] and semiconductor bulk materials containing a high density of deep-level impurities [13-15], amongst others. This work deals exclusively the first of these (QD-IBSCs), paying specific attention to the InAs/GaAs based prototypes, which have received particular attention.

Research into IBSCs has largely been conducted in two phases; the earliest works were theoretical studies of the thermodynamic consistency [16] and absolute limiting efficiency [1] of the IBSC concept, and theoretical studies of possible implementations and design issues $[3,17,18]$. In the second phase, extensive work was undertaken to fabricate and develop QD-IBSC prototypes [4-8] and demonstrate that they fulfill the fundamental operating principles of the IBSC: most importantly the generation of subbandgap photocurrent due to sequential two-photon absorption via the intermediate band/levels [19], and a $V_{o c}$ that is above the subbandgap absorption thresholds [20]. A recent review of which technologies have demonstrated these principles can be found in Ref. [21]; in the context of QD-IBSCs, they have been demonstrated

\footnotetext{
* Corresponding author. Tel.: +44 207594 1030; fax: +44 2075942077.

E-mail address: a.mellor@imperial.ac.uk (A. Mellor).
}

on the InAs/GaAs material system, though the voltage preservation has only been demonstrated at low temperatures [20].

Following the demonstration of the operating principles, QDIBSC research is now entering a third phase, whose ultimate goal must be to deliver solar cells with conversion efficiencies surpassing those of conventional single-gap devices. For this to be achieved, two broad technological advances are needed. First, QD-IBSCs must demonstrate high $V_{o c} s$ at room temperature. This requires that thermal carrier escape from the QDs be suppressed [22] and that non-radiative processes in the cells be minimised. Recent results of reduced thermal escape due to a higher bandgap (AlGaAs) host material [23], and high QD-IBSC $V_{o c}$ s due to improved material quality [24] are promising in this regard. Second, the subbandgap photocurrent must be improved significantly by increasing subbandgap photon absorption in the QD array. This may be achieved by increasing the number of QDs, by optical absorption enhancement, or a combination of both. QD arrays are being fabricated with an increasing number of QDs both in the context of QD-IBSCs [25] and elsewhere [26]. Optical absorption enhancement is standard in wafer-based crystalline silicon solar cells $[27,28]$ and thin-film crystalline silicon solar cells [29], and we expect and encourage researchers to bring this technology into the context of QD-IBSCs.

This paper is aimed at informing this third phase of research. The experimental achievements described in the previous paragraphs have been accompanied by theoretical studies of the fabricated prototypes [7,30-34], giving us a much deeper understanding of their operation and highlighting pathways to improvement. Based on this understanding, it is now possible to make a realistic assessment of the feasibility of achieving high efficiencies 
using InAs/GaAs QD arrays, and to quantify the advances that are required to do so. In this paper, such an assessment is made using our recently developed realistic detailed balance model, which has been shown to reproduce well the measured quantum efficiency of an InAs/GaAs QD-IBSC prototype and its temperature dependence [34].

This paper has three objectives:

1. to reassess the feasibility of achieving high efficiencies in InAs/ GaAs QD-IBSCs, based on the understanding that has been acquired over the previous years.

2. to quantify the degree of absorption enhancement that is required for present QD-IBSCs to supersede the efficiency of an equivalent single gap reference.

3. to compare the relative merits of absorption enhancement via increasing the number of QDs and employing optical absorption enhancement techniques.

\section{The detailed balance model applied to the QD-IBSC prototype}

The subject of our study is the sample labelled SB in Ref. [22] and S3 in Ref. [35]. This sample has been chosen since it has demonstrated all of the operating principles of the IBSC concept [21]. A simplified level/band diagram of a single QD in this exemplary QD-IBSC is shown in Fig. 1. The grey lines represent the $\mathrm{VB}$ and $\mathrm{CB}$ band edges, the offsets being due to the InAs QD. The confining potential in the $\mathrm{CB}$ supports three discrete bound state levels in the energy range of the host forbidden band. These are denoted IB levels, and are labelled IB1, IB2 and IB3, with energies $E_{I B 1}, E_{I B 2}$ and $E_{I B 3}$, respectively (these correspond to the states labelled (1 111 ) , ( $\left.\begin{array}{lll}2 & 1 & 1\end{array}\right) /\left(\begin{array}{lll}1 & 2 & 1\end{array}\right),\left(\begin{array}{lll}2 & 2 & 1\end{array}\right)$ in Ref. [31]). The VB potential pedestal supports a quasi-continuum of confined heavyhole states which act to displace the energy onset of the VB to $E_{V B \text {, eff }}$ (shown), thus reducing the effective overall bandgap from that of the GaAs host $(1.42 \mathrm{eV})$ to the value $E_{g,}$ eff $=1.24 \mathrm{eV}$. The energies of the principal transitions between the VB pedestal and the IB levels, $E_{t 1}$, $E_{t 2}, E_{t 3}$, are given in the figure.

The system is treated as five distinct electron populations: that of the $\mathrm{CB}$, that of the VB (whose upper energy onset is considered

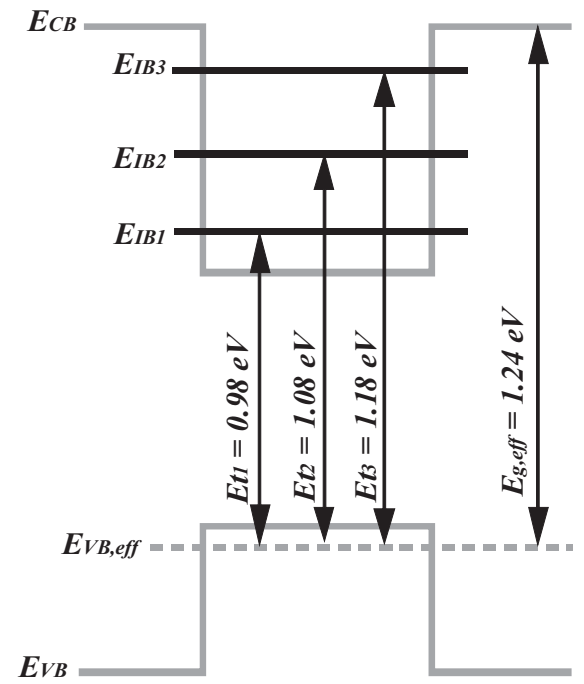

Fig. 1. A simplified band diagram of a single QD in the exemplary QD-IBSC. Upper grey line: conduction band edge. Lower grey line: valence band edge. Black lines: confined state energy levels whose energy is within the host forbidden band. Dashed grey line: effective valence band edge. to be at $E_{V B}$, eff), and those of the three discrete IB energy levels. The electron populations are each described by a Fermi-Dirac function with a distinct quasi Femi level (QFL): $E_{F, I B 1}, E_{F, I B 2}$ and $E_{F, I B 3}$, $E_{F, C B}$ and $E_{F, V B}$.

The model is based on solving five simultaneous equations to yield the five QFLs defined above. Three equations emerge from the condition that the electron population in each IB level is time constant, implying that the sum of transitions received by any IB level per second is equal to the sum of transitions proceeding from that level. One equation emerges from assuming that the net charge is zero in most of the QD layer stack. The final equation sets the difference between the VB and CB QFLs equal to the externally applied bias voltage, which is equivalent to assuming infinite carrier mobility throughout the device. This is approximately achieved in GaAs solar cells. Present QD-IBSC prototypes exhibit higher series resistance [36]. However, this is likely due to unwanted recombination and non-optimal contacting, both of which can be improved. Indeed, IV curves of more recent QDIBSC prototypes suggest series resistances close to GaAs references have been achieved [24].

Once calculated, the QFLs are then used to calculate the strengths of the subbandgap transition currents. Adding these to the above-bandgap photocurrent, which is calculated using the standard single-gap Shockley-Queisser model with the bandgap of the $\mathrm{GaAs}$ host $(1.42 \mathrm{eV})$, yields the total current produced at the terminals of the QD-IBSC for a given applied voltage.

The realistic detailed balance model differs from previous detailed balance models used to calculate the upper limits to IBSC efficiency [1] in two important aspects. First, whereas previous detailed balance models assume that the subbandgap transitions absorb all incident photons in the corresponding energy range, the present model calculates the subbandgap transition currents using the absorption coefficients that have been calculated for the investigated QD-IBSC using a four-band $\mathrm{k} \times \mathrm{p}$ model [32,37]. This $\mathrm{k} \times \mathrm{p}$ model has been shown to have good agreement with the measured absorption [32]. Second, the model considers the multiple intermediate levels, and not just the ground state. This is important since the thermal escape of electrons from the QD has been shown to occur via the ladder of excited states between the ground state and the $\mathrm{CB}[33,34]$. Correspondingly, we expect the $V_{o c}$ to be limited by recombination that occurs via the excited levels.

It must be emphasised that the model only considers radiative processes (i.e. photogeneration and radiative recombination). In our previous work, the model was shown to reproduce well the experimental quantum efficiency, and its temperature dependence, at short circuit: a condition under which the prototype is believed to be radiatively dominated. Closer to open circuit, the present prototypes are believed to be dominated by non-radiative processes, such as Shockley-Read-Hall recombination. Therefore, we expect the model to overestimate the $V_{o c}$ of the present prototypes (a simple comparison of the results presented here with experimental $V_{o c} S$ in Ref. [20] verifies this). Nonetheless, the use of this radiative model is justified for the present study, as is described in the following.

QD-IBSCs must be made to be radiatively dominated under operating conditions if they are to supersede the efficiency of single-gap devices. The purpose of this paper is to determine whether, using the present $\mathrm{QD}$ and host materials and QD dimensions, radiative operation combined with absorption enhancement is sufficient for these efficiencies to be achieved. It is therefore appropriate to use a radiative model. Notice that the recombination involved in the radiative model is unavoidable and therefore it represents its lower limit; non-radiative recombination is deemed to be avoidable.

A detailed description and derivation of the model can be found in Ref. [34]. A brief mathematical description is given in 
Appendix A of the present paper for the convenience of the reader The model has been adapted to allow for higher subbandgap absorption. The adaption is derived in Appendix B.

\section{Means of increasing the subbandgap photon absorption}

Two means of increasing the absorption are investigated. The first is by increasing the number of QDs in the QD stack. The second is by so-called light trapping, in which optical texturing of the substrate causes incident photons to be more strongly absorbed in the QD stack. Both means are described in the following paragraphs, along with the effects they are expected to have on the subbandgap photocurrents.

\subsection{Increasing the number of quantum dots}

The overall subbandgap absorption can be increased by increasing the overall QD density per $\mathrm{cm}^{2}$ of solar cell area. This can be achieved by increasing the areal QD density per layer and/ or by increasing the number of QD layers. The density per $\mathrm{cm}^{3}$ is also increased if we reduce the separation between layers so permitting the reduction the IB thickness. The IB region of the studied prototype is a layer stack of 30 QD layers, each layer having an areal QD density of $4 \times 10^{10} \mathrm{~cm}^{-2}$ [22]. More recently, QD stacks have been grown with more layers and other have been grown with higher areal QD densities per layer. Ref. [25] reported an InAs/GaNAs QD stack with 100 layers and an areal QD density of $4 \times 10^{10} \mathrm{~cm}^{-2}$, and Ref. [26] reported an InAs/GaNAs QD stack with 300 layers and an areal QD density of $6 \times 10^{10} \mathrm{~cm}^{-2}$. These represent an increase in the number of layers of a factor of 3 and 10, respectively, compared to our prototype. Other authors have reported InAs/GaAs QDs with an areal density per layer of $1.1 \times 10^{11} \mathrm{~cm}^{-2}$ [38] and GaAsSb/GaAs QDs with an areal density per layer of $4 \times 10^{11} \mathrm{~cm}^{-2}$ [39]: increases by factors of 3 and 10 respectively. In these works, only a few QD layers were grown, making the overall density similar to our prototype; however, the areal densities per layer are remarkable.

Growing a high number of layers and achieving a high QD density per layer simultaneously will undoubtedly require further development. However, based on these results in the literature we can envisage the combination of a 10 fold increase in areal QD density per layer and a 10 fold increase in the number layers, leading to an overall increase in the number of QDs by a factor of 100. Further increases can be expected beyond this as technology develops in the future.

\subsection{Absorption enhancement via light trapping}

The probability that an incident photon is absorbed in a medium is proportional to the distance it travels in that medium. This distance can be increased without increasing the layer thickness by employing so-called light trapping or light confinement techniques. These involve the incorporation of optical components into the solar cell with the aim of increasing the optical path length of incident photons within the absorber layer. Light trapping techniques may involve a rear reflector, a scattering mechanism, or both. A rear reflector causes photons not absorbed on making a single pass of the absorber layer to be reflected and make another pass before leaving the cell, thus increasing their probability of absorption. Rear reflectors include metals, dielectric Bragg-type reflectors [40] and even white paint [41]. Scattering deflects incident light into oblique directions. These have higher path lengths in the absorber layer than light which traverses normally. What's more, light deflected at certain angles can be confined in the solar cell by total internal reflection at the surface, causing it to make multiple passes of the absorber layer before escape. Scattering mechanisms include roughened surfaces [42], geometrically textured surfaces [43], diffraction gratings [44] and metal or dielectric nanoparticles [45].

It has been shown by Yablonovitch that, in the limit of completely isotropic scattering and a perfect rear reflector, the mean optical path length enhancement of incident photons approaches $4 n^{2}$ : the so called Lambertian limit. In this work, we have taken the refractive index of the GaAs wafer substrate and InAs/GaAs QD stack to be $n=3.5$, making the Lambertian limit a factor of around 50. In practise, more light tends be transmitted or reflected specularly than is scattered. Consequently, the surface textures used in present solar cells are believed to offer belowLambertian light trapping, with optical path length enhancements of around 10 [46]. It should be observed that the Lambertian Limit is not an upper limit; it can be superseded at some wavelengths through the use of diffraction gratings [47], angle-selective filters [48] and near-field absorption enhancement techniques [49]. However, in this work, we take an optical absorption enhancement of 50 to be optimistic and absorption enhancements of 10 to be realistic, and do not consider the wavelength dependence of the absorption enhancement.

\subsection{The effect of absorption enhancement on the subbandgap transition currents}

As described in Appendix A and B, the subbandgap photon absorption is characterised by the thickness of the QD stack, $W$, and by two absorption coefficients $\alpha_{x, y}$ and $\alpha_{i s o}$, which respectively describe the absorption of photons polarized in the $x, y$ plane (those incident from the sun) and the absorption of isotropically polarized photons (thermal photons incident from the surroundings and the substrate). The two means of increasing the subbandgap photon absorption, described above, affect the subbandgap transition currents in different ways. The effect of increasing the number of QDs is to increase the absorbance $\left(\alpha_{x, y} W\right.$ and $\left.\alpha_{i s o} W\right)$ by a constant factor, which we denote $\Lambda_{Q D}$. This is true whether the number of QDs is increased by increasing the number of layers or by increasing the areal QD density per layer. Note that increasing the number of QDs therefore affects both the illumination- and dark currents. The effect of employing light trapping is quite different. Photons incident from the sun are scattered by the scattering mechanism, making them more isotropic, and thus increasing their optical path length inside the absorber layer. However, thermal and luminescent photons are already isotropic before reaching the surface texture. Light trapping is therefore expected to affect the illumination current, but not the dark current. To summarize, the detailed balance model is adapted to incorporate the effect of absorption enhancement by using the following expressions for the illumination and dark currents for each subbandgap transition ( $l$ is the lower level and $u$ the upper level; see Appendix A for a definition of other terms).

$$
\begin{aligned}
& J_{l \rightarrow u}^{L}=q_{e} \int_{0}^{E_{g}} d(\hbar \omega)\left(1-\exp \left[-\Lambda_{o p t} \Lambda_{Q D} \alpha_{x, y} W\right]\right) \Phi_{i n c} \\
& J_{l \rightarrow u}^{D}=q_{e} \int_{0}^{E_{g}} d(\hbar \omega) \frac{2 \pi n^{2}(\hbar \omega)^{2}}{h^{3} c^{2}}\left(f_{B, \mu T_{c}}-f_{B, 0 T_{c}}\right) I^{D}\left[\Lambda_{Q D} \alpha_{i s o} W\right]
\end{aligned}
$$

where $\Lambda_{O D}$ is the factor by which the overall QD density has been increased per $\mathrm{cm}^{2}$ of solar cell area (not per QD layer) with respect to the studied prototype, and $\Lambda_{o p t}$ is the mean optical path length enhancement of incident photons achieved by light trapping. Note that $\Lambda_{\text {opt }}$ only appears in $J^{L}$, whereas $\Lambda_{O D}$ appears in both. From this insight, it should already be clear that absorption enhancement via 
light trapping will lead to a better efficiency boost than via increasing the number of QDs. This is examined quantitatively further on.

\section{Results}

\subsection{Reference curves}

The purpose of this work is to predict if and how present QDIBSC prototypes can be made to supersede the efficiency of stateof-the-art single-gap solar cells. It is therefore necessary to compare the results to the Shockley-Queisser limit [2] for singlegap solar cells. Using the standard Shockley-Queisser model $[2,50]$, we have calculated that the maximum single-gap efficiency is $34.2 \%$ and is achieved with a bandgap of $E_{g}=1.17 \mathrm{eV}$. This has been calculated assuming the same set of conditions assumed for the QD-IBSC: $1000 \times$ concentration, $300 \mathrm{~K}$ cell temperature and an étendue [51] of escaping luminescence proportional to $1+n^{2}$, the 1 representing escape into the vacuum at the front and the $n^{2}$ into the electrically inactive wafer substrate at the rear. The JV characteristics of this hypothetical limiting single-gap device are shown in Table 1 under the row heading "SQ single-gap limit". It should be observed that, where we have made idealised assumptions in calculation the QD-IBSC current (infinite carrier mobility etc.), the same assumptions have been made for the singlegap-limit solar cell.

In addition, we take as a second reference an ideal IBSC with a single intermediate level and the same principal bandgaps as the QD-IBSC under investigation (that is with $E_{H}=E_{t 1}$ and $E_{L}=E_{g, e f f}-E_{t 1}$ ). The $J V$ curve for the ideal IBSC is calculated by applying the model in Ref. [1]; full absorption of incident photons and the radiative limit are assumed. The $J V$ characteristics and bandgaps are shown in Table 1 under the row heading "IBSC limit". Observe that this is not the absolute limit to IBSC efficiency, since non-optimal bandgaps have been selected to correspond to the QD-IBSC prototype.

The JV characteristics of the QD-IBSC prototype, calculated using the realistic detailed balance model, are also shown in Table 1.

\subsection{JV characteristics of QD-IBSCs with increased subbandgap absorption}

$J V$ curves have been calculated for the QD-IBSC cell taking different combinations of $\Lambda_{o p t}$ and $\Lambda_{Q D}$ in order to predict the effect of increasing the number of QDs, employing light trapping, or a combination of both. The short circuit current density, $J_{s c}$, open circuit voltage, $V_{o c}$, and conversion efficiency, $\eta$, have been extracted from these curves, and are plotted in Fig. 2 (top, middle and bottom respectively). Each curve is plotted as a function of the $\mathrm{QD}$ number enhancement factor, $\Lambda_{\mathrm{QD}}$, and different curves are plotted for different values of the optical path length enhancement due to light trapping, $\Lambda_{\text {opt }}$. Results are plotted for $\Lambda_{\text {opt }}=1, \Lambda_{\text {opt }}=10$, and $\Lambda_{\text {opt }}=50$, which respectively represent no light trapping, realistic light trapping using presently employed surface textures,
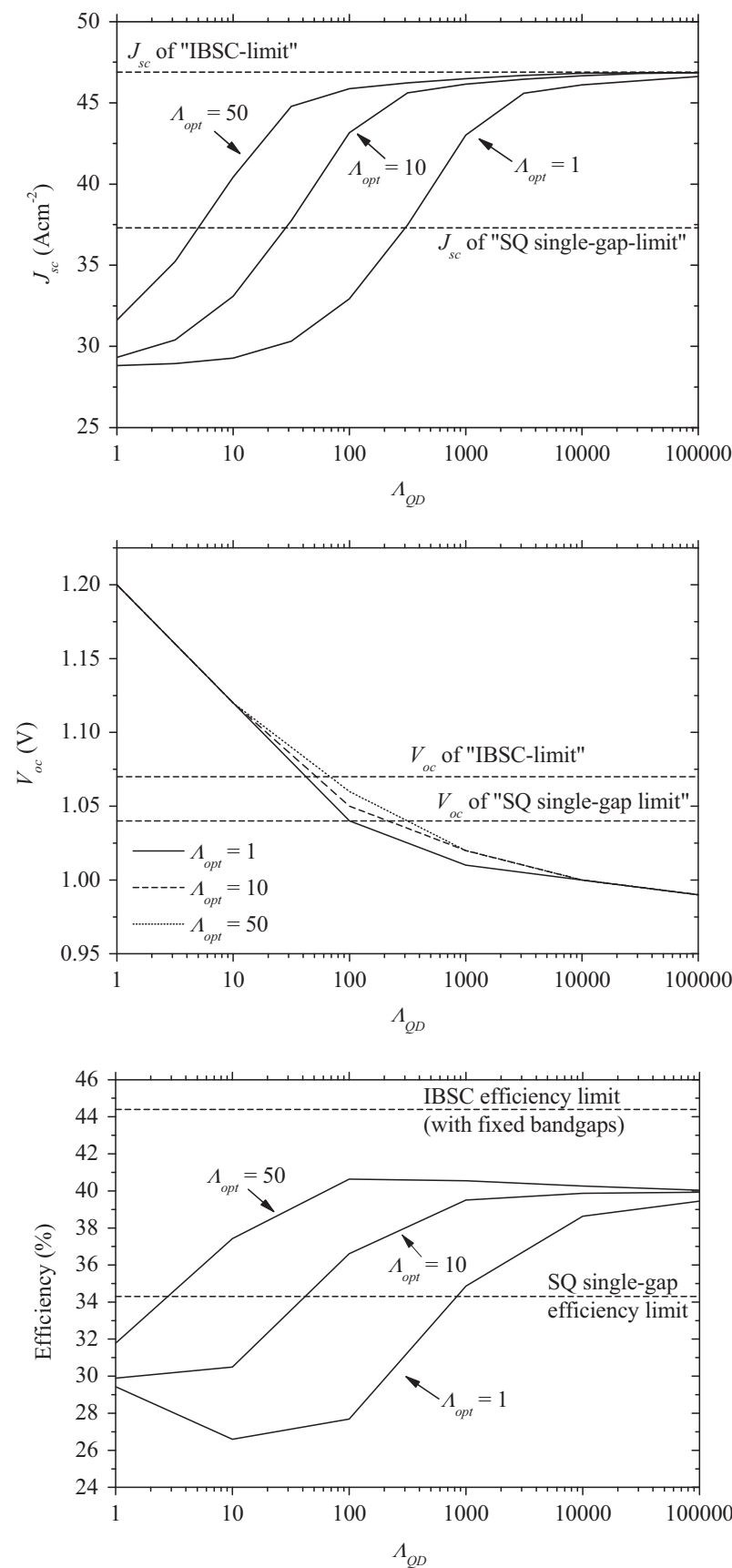

Fig. 2. $J V$ characteristics of the QD-IBSC prototype for different absorption enhancements. $\Lambda_{Q D}$ is the factor by which the number of QDs per unit solar cell area is increased compared to the present prototype. $\Lambda_{\text {opt }}$ is the optical path length enhancement achieved by light trapping. Top: $J_{s c}$; Middle: $V_{o c}$; Bottom: conversion efficiency. In each graph, the labelled horizontal lines represent values for the single-gap limit and the IBSC limit. The reader is reminded that the single-gap-limit is for a bandgap of $E_{g}=1.17 \mathrm{eV}$, which maximises the efficiency. The IBSC limit has bandgaps corresponding to the principal bandgaps of the QD-IBSC prototype, and is therefore not the absolute upper limit to IBSC efficiency.

Table 1

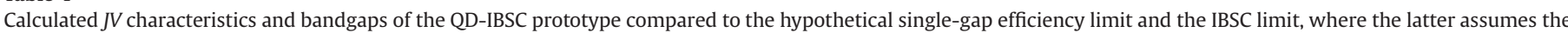
same principal bandgaps as the prototype.

\begin{tabular}{|c|c|c|c|c|c|c|}
\hline & $E_{g} / \mathrm{eV}$ & $E_{H} / \mathrm{eV}$ & $E_{L} / \mathrm{eV}$ & $J_{s c} / \mathrm{A} \mathrm{cm}^{-2}$ & $V_{o c} / \mathrm{V}$ & Efficiency (\%) \\
\hline QD-IBSC prototype & \multicolumn{3}{|c|}{ see Table A1 (Appendix A) for energy levels } & 28.9 & 1.18 & 29.4 \\
\hline SQ single-gap limit & 1.17 & $\mathrm{n} / \mathrm{a}$ & $\mathrm{n} / \mathrm{a}$ & 37.3 & 1.04 & 34.3 \\
\hline IBSC limit & 1.24 & 0.98 & 0.26 & 46.9 & 1.07 & 44.4 \\
\hline
\end{tabular}


and light trapping at the Lambertian limit. In each graph, the horizontal lines represent the respective quantity for the SQ single-gap limit, as well as for the ideal IBSC limit described previously and shown in Table 1.

Looking at the $J_{s c}$, we can see that, without light trapping, the number of QDs must be increased by a factor of around 300 for the QD-IBSC $J_{s c}$ to exceed that of the single-gap-limit solar cell. If absorption enhancements by factors of 10 and 50 are achieved via light trapping, this value drops to 30 and 5 respectively.

The behaviour of the $V_{o c}$ requires careful discussion. First, the model predicts that, under radiative conditions, the $V_{o c}$ of the QDIBSC with no absorption enhancement is higher than that of the ideal QD-IBSC. This appears to be a confusing result at first. The chosen ideal IBSC has an overall bandgap of $E_{g}=1.24 \mathrm{eV}$; this being

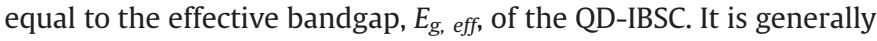
supposed that the QD-IBSC's $V_{o c}$ is limited at most by its effective bandgap, or, perhaps more likely, by one of the lower subbandgap transition energies $\left(E_{t 1}, E_{t 2}\right.$ or $E_{t 3}$ in Fig. 1). We would therefore expect the $V_{o c}$ of the QD-IBSC to be lower or equal to that of the ideal IBSC. However, since the subbandgap photon absorption/ emission is so weak, and radiative conditions are assumed, the recombination via subbandgap transitions (those from the $C B$ to the VB pedestal included) is extremely weak. This is to be compared to the above-bandgap photocurrent generated in the GaAs emitter (around $29 \mathrm{~A} \mathrm{~cm}^{-2}$ ), which absorbs all incident photons above the GaAs bandgap (1.42 eV). Hence, in the absence of absorption enhancement, and in the radiative limit, the $V_{o c}$ of the QD-IBSC is more similar to that of a single-gap GaAs device (around $1.26 \mathrm{~V}$ under the assumptions of the SQ model). Once again, the reader is reminded that present QD-IBSCs are dominated by non-radiative recombination, and therefore have much lower $V_{o c} s$ (around $0.9 \mathrm{~V}$ for the prototype on which this study is based at $300 \mathrm{~K}$ and $1000 \times[20])$. It should be mentioned that the fact that a high $V_{o c}$ is predicted for a weakly absorbing solar cell is of no practical use. A highly efficient solar cell must absorb most incident photons; hence, only the results for stronger absorption are of practical importance.

We can see that there is a steady drop in the QD-IBSC's $V_{o c}$ as the number of QDs increases. This follows from our discussion in the previous paragraph. As the number of QDs is increased, the total current becomes more strongly influenced by the subbandgap recombination currents, and the overall $V_{o c}$ falls to a lower value. Importantly, the $V_{o c}$ is predicted to fall to a value below that of the aforementioned IBSC limit, meaning that the $V_{o c}$ is limited not by the effective bandgap, but by one of the lower energy gaps $\left(E_{t 1}, E_{t 2}\right.$ or $\left.E_{t 3}\right)$. This is due to the CB QFL being pinned to one of the IB levels at open circuit at room temperature.

We can confirm from the calculations that, for high values of $\Lambda_{\mathrm{OD}}$, the CB QFL is at around $0.16 \mathrm{eV}$ below the CB onset, meaning that it is pinned at the IB2 level. This follows naturally from our results in Refs. [33,34], in which it was shown that there is a strong radiative thermal escape from the IB levels to the $C B$, but that this radiative escape always occurs via the IB2 level. Since the CB QFL is pinned to the IB2 level, the $V_{o c}$ is limited by $E_{t 2}$. This is higher than the absorption onset, which is at $E_{t 1}$, and consequently the SQ limit can be superseded. However, it is lower than the effective bandgap, $E_{g, e f f}$, and, consequently, the QD-IBSC $V_{o c}$ tends to a value below that of the IBSC limit. It shall be seen later on that the conversion efficiency correspondingly levels off at a value between the SQ limit and the IBSC limit.

The $V_{o c}$ is mainly affected by the dark current, and therefore does not vary significantly with optical path length enhancement, although there is a slight improvement of the $V_{o c}$ for higher $\Lambda_{\text {opt }}$. This is probably related to the increased $J_{s c}$, which is analogous to the way in which the $V_{o c}$ of a solar cell tends to improve logarithmically with increased concentration.
The most important metric resulting from the $J V$ curve is the conversion efficiency. In the absence of light trapping $\left(\Lambda_{\text {opt }}=1\right)$, the predicted efficiency actually drops on increasing $\Lambda_{\mathrm{QD}}$ from 1 to 10 ; this is due to the predicted drop in $V_{o c}$. The efficiency then rises on increasing $\Lambda_{\mathrm{QD}}$ and surpasses the SQ single-gap limit for an enhancement of $\Lambda_{Q D}>1000$. On further increasing $\Lambda_{Q D}$, the efficiency saturates at about $40 \%$, which is between that of the single-gap reference and the ideal IBSC. This follows from our previous assertion that the $V_{o c}$ is limited by $E_{t 2}$ and not $E_{t 1}$ or $E_{g, e f f}$.

Clearly, an increase in the number of QDs by a factor of 1000 or more is far beyond present technological capabilities. However, we can see from Fig. 2 (bottom) that, by employing light trapping, a much lower increase in the number of QDs are required for the QD-IBSC to supersede the single-gap limiting efficiency. For optical enhancements of $\Lambda_{o p t}=10$ and $\Lambda_{o p t}=50$, an increase in the number of QDs by factors of $\Lambda_{Q D}=30$ and $\Lambda_{Q D}=3$ are required respectively.

Comparing Fig. 2 top and bottom, we can see that absorption enhancement due to light trapping has a more profound effect on the efficiency than on the $J_{s c}$. To illustrate this, $J V$ curves have been calculated for different combinations of $\Lambda_{Q D}$ and $\Lambda_{\text {opt }}$ that lead to an overall absorption enhancement of 1000 -the overall absorption enhancement being the product $\Lambda_{\mathrm{QD}} \times \Lambda_{\text {opt }}$. The combinations are $\left(\Lambda_{o p t}=1, \Lambda_{Q D}=1000\right),\left(\Lambda_{o p t}=10, \Lambda_{Q D}=100\right)$ and $\left(\Lambda_{o p t}=50\right.$, $\left.\Lambda_{Q D}=20\right)$. The three $J V$ curves are plotted in Fig. 3. All have the same $J_{s c}$, as would be expected. However, the $V_{o c}$ is higher when a greater proportion of the enhancement is achieved by light trapping. Hence, the advantage of light trapping is two-fold; it reduces the number of QDs that are required for a high $J_{s c}$, making the QD stack more technologically feasible, and it also allows this high $J_{s c}$ to be accompanied by a higher $V_{o c}$ than would possible if no light trapping were employed.

\section{Conclusions}

The JV characteristics of an InAs/GaAs QD-IBSC have been calculated using the realistic detailed balance model. The model is used to predict the $J_{s c}, V_{o c}$ and overall efficiency that would be expected if non-radiative processes were supressed and photon absorption were enhanced by different degrees, either by increasing the number of quantum dots, employing light trapping or a combination of the two. Calculations are made assuming $1000 \times$ sunlight concentration and $300 \mathrm{~K}$ operating temperature. Results are compared to the Shockley-Queisser limits for single-gap devices under the same conditions, as well as to the limiting

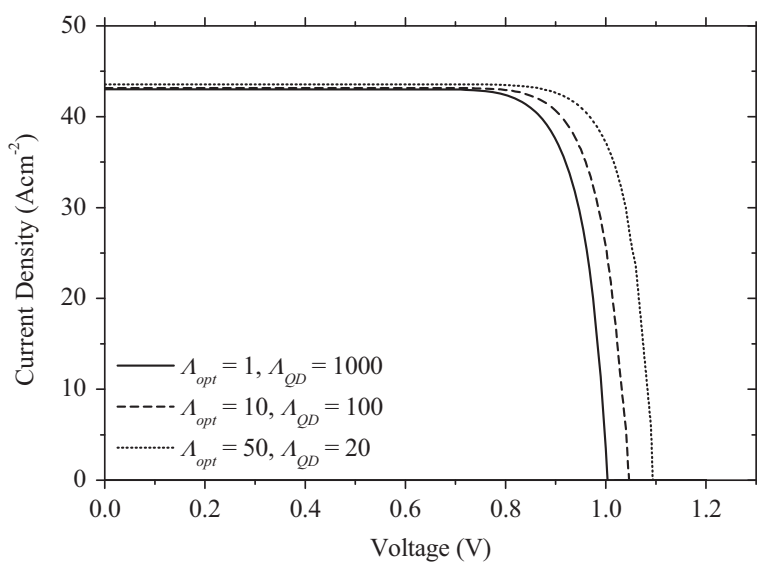

Fig. 3. $J V$ curves for different combinations of $\Lambda_{Q D}$ and $\Lambda_{\text {opt }}$. All three combinations give a total absorption enhancement of $\Lambda_{Q D} \times \Lambda_{o p t}=1000$. 
efficiency of an ideal IBSC with the same principle bandgaps as the studied prototype.

On increasing the number of QDs indefinitely, the efficiency saturates at a value below the IBSC limit. This is because the $V_{O C}$ is limited due to the thermal escape from the IB levels to the CB [22], which has been revealed in previous works to have a strong radiative component $[33,34]$. However, the QD-IBSC efficiency is predicted to supersede the single-gap Shockley-Queisser limit. This is because, under radiative conditions, the $V_{o c}$ is predicted to be limited by a higher energy gap than the absorption onset.

In the absence of light trapping, a factor of 1000 increase in the number of QDs, compared to the studied prototype, is required for the QD-IBSC to supersede the SQ limit. If optical path length enhancements of 10 and 50 are achieved by light confinement techniques, this value drops to 30 and 3 respectively. We believe that combing an increase in the number of QDs by a factor of 30 with an optical absorption enhancement of 10 is within present capabilities, or at least those expected in the near future.

Finally, it has been argued that absorption enhancement via light trapping is especially beneficial since it is expected to affect only the illumination current and not the dark current. Of course, both light trapping and an increase in the number of QDs will be required.

We hope that researchers will see these results as promising and a motivation to make the final advances necessary to achieve high efficiency QD-IBSCs.

\section{Acknowledgements}

This work has been supported by the European Commission through the project NGCPV (Grant 283798) and by the Spanish National Research Program PROMESA (ENE2012-37804-C02-01).

\section{Appendix A. summary of calculation method and input parameters}

Here, a brief description of the detailed balance model, first derived in Ref. [34], is given, along with the input parameters for the QD-IBSC under investigation.

Generation and recombination currents exist between the five electronic populations due to the respective emission and absorption of photons. The net generation current (generation or upward traffic of electrons up minus recombination or downward traffic) between a given pair of bands/levels is denoted $J_{l \rightarrow u}$, where $l$ and $u$ are respectively the lower and upper level or band. This can be conveniently decomposed into an illumination current, $J_{l \rightarrow u}^{L}$, and a dark current, $J_{l \rightarrow u^{*}}^{D}$

$J_{l \rightarrow u}=J_{l \rightarrow u}^{L}+J_{l \rightarrow u}^{D}$

where

$J_{l \rightarrow u}^{L}=q_{e} \int_{0}^{E_{g}} d(\hbar \omega)\left(1-\exp \left[-\alpha_{x, y} W\right]\right) \Phi_{i n c}$

$J_{l \rightarrow u}^{D}=q_{e} \int_{0}^{E_{g}} d(\hbar \omega) \frac{2 \pi n^{2}(\hbar \omega)^{2}}{h^{3} c^{2}}\left(f_{B, \mu T_{c}}-f_{B, 0 T_{c}}\right) I^{D}\left[\alpha_{i s o} W\right]$

$I^{D}[s]=\pi\left(1-(1-2 s) \exp [-2 s]+4 s^{2} E i[-2 s]\right)$

$E i[s]=-\int_{-s}^{\infty} d t \frac{e^{-t}}{t}$

$\hbar \omega$ is the photon energy, $\Phi_{i n c}[\hbar \omega]$ is the incident photon flux per unit time and area, $n$ is the refractive index of the QD layer stack, $q_{e}, h$ and $c$ have their usual meanings, $s$ and $t$ are auxiliary free variables, $f_{B, \mu T_{c}}$ is the Bose-Einstein energy distribution of luminescent photons at temperature $T_{c}$ and chemical potential $\mu=E_{F, u}-E_{F l}$, and $f_{B, 0 T_{C}}$ denotes the Bose-Einstein distribution at zero chemical potential. $f_{B, \mu T_{C}}$ and $f_{B, 0 T_{C}}$ represent respectively the populations of luminescent and thermal photons; these respectively escape into and are incident from the air at the front surface and the electrically inactive substrate at the rear.

It should be observed that the transition currents between two levels were, in our previous paper [34], derived in the limit of low absorption. In this work, we consider the effects of increasing photon absorption in the QD layer stack. The currents shown in Eq. (A2) have therefore been adapted to allow for the possibility of higher absorption; i.e., they have been generalised. The adaption to the derivation that yields Eq. (A2) is given in Appendix B of the present paper.

$\alpha_{x, y}[\hbar \omega]$ is the absorption coefficient of the QD stack averaged over photon polarizations in the $x y$ plane (as is the case for photons incident from the sun, to a good approximation). $\alpha_{i s o}[\hbar \omega]$ is the absorption coefficient of the QD stack averaged over all polarizations for photons incident isotropically incidence (as is the case for thermal photons incident from the surroundings or the substrate at $T_{C}$ ). Both $\alpha_{x, y}$ and $\alpha_{i s o}$ are dependent on the Fermi filling factors of the lower and upper levels (denoted $f_{u}$ and $f_{l}$ respectively), which are in turn dependent on the unknown quasi Fermi levels. It is therefore convenient to define the quantities $\alpha_{x, y}^{\max }$ and $\alpha_{i s o}^{\max }$ by

$\alpha_{x, y}^{\max }=\alpha_{x, y} /\left(f_{l}-f_{u}\right)$

$\alpha_{i s o}^{\max }=\alpha_{i s o} /\left(f_{l}-f_{u}\right)$

$\alpha_{x, y}^{\max }$ and $\alpha_{i s o}^{\max }$ are independent of the quasi Fermi levels and therefore serve as the input for the detailed balance model; they have been calculated for the QD-IBSC prototype in Refs. [31,32,52-54], and are plotted in Fig. A1.
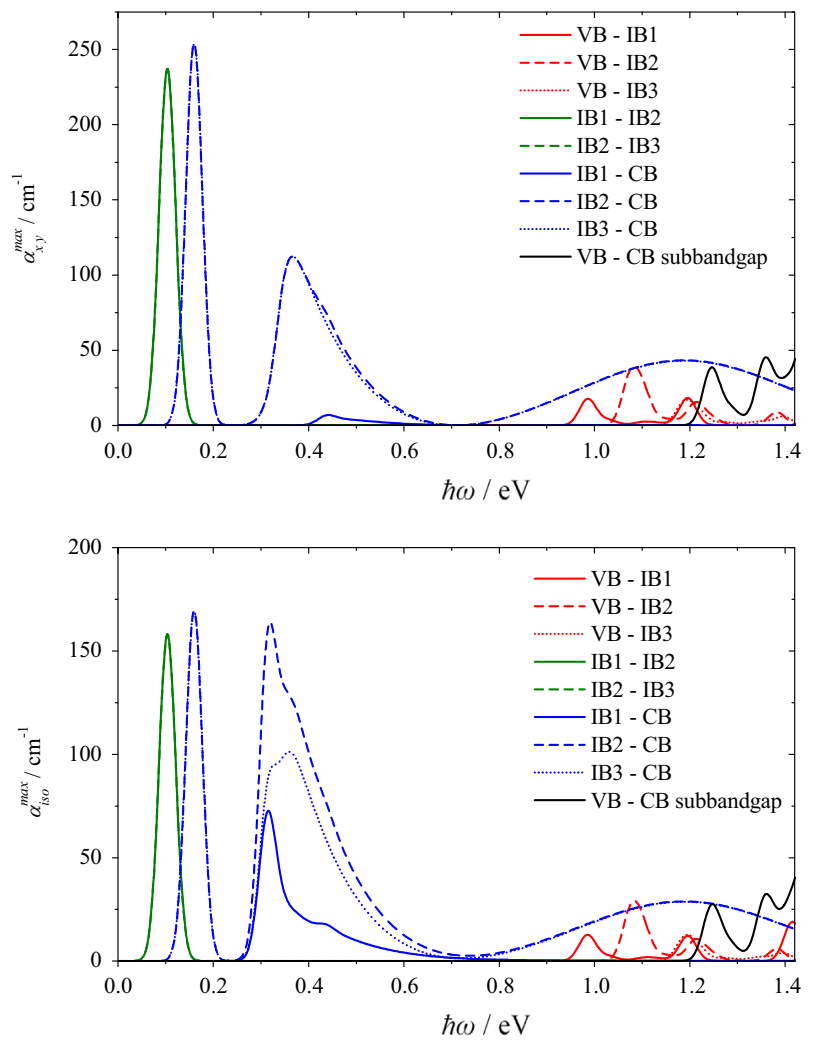

Fig. A1. Absorption coefficients for the different transitions in the QD-IBSC prototype before modification by the electron occupancies of the lower and upper levels. Top: absorption coefficients for photons polarized in the $x y$ plane. Bottom: absorption coefficient for isotropic photons. Each curve represents a different electronic transition. Curves are shown for all pairs of bands and levels shown in Fig. 1, except for the IB1-IB3 transition, which is forbidden [31]. 
The detailed balance model is based on solving five simultaneous equations. The first three are the continuity of the net transition currents that populate and depopulate each IB level. Due to the conventional GaAs layers on either side of the QD stack, current can only be extracted via the VB and $\mathrm{CB}$ and not directly from the IB levels. Hence, for each IB level with index $k$, we can write a current continuity equation:

$\sum_{l} J_{l \rightarrow k}-\sum_{u} J_{k \rightarrow u}=0$

where the first and second sums are made over all levels (or bands) whose energy is respectively lower or higher than that of IB level $k$. Eq. (A4) presents three equations; one for each intermediate band level.

Charge neutrality is assumed to in the QD stack. This condition is found in the inside (far from the edges) of thick stacks of QDs, as is the case for the QD-IBSC under investigation [55]. We can therefore write the equation

$n_{C B}-p_{V B}+n_{I B}-N_{d}^{+}=0$

where $n_{C B}$ and $p_{V B}$ are the concentrations of electrons and holes in the $\mathrm{CB}$ and $\mathrm{VB}$ respectively (taking also the confined hole states into account), $n_{I B}$ is the electron concentration in the several IB states (summed), and $N_{d}^{+}$is the concentration of ionized donor atoms used to prefill the IB.

Finally, we make the simplifying assumption that the terminal voltage is equal to the split between the VB and CB QFLs. This is equivalent to assuming infinite carrier mobility and, therefore, zero series resistance. The appropriateness of this assumption is discussed in the introduction.

$E_{F, C B}-E_{F, V B}=q_{e} V$

Through Eqs. (A4)-(A6), we have a system of simultaneous equations with as many equations as unknowns (five in the example analysed here). The input parameters are the energy levels of both confined and extended states in all bands (calculated in Ref. [31] ), the Fermi-level-free absorption coefficients ( $\alpha^{\max }$ ) for all possible transitions (shown in Fig. A1), the cell temperature, the terminal voltage and the illumination photon flux. The problem is solved numerically using Wolfram Mathematica ${ }^{\circledR}$, yielding the QFLs. The net generation current for each transition is then calculated using Eqs. (A1) and (A2).

The incident photon flux $\Phi_{\text {inc }}\left[E_{\text {phot }}\right]$ is taken to be that directly incident on the solar cell from the sun, which is modelled as a black body at temperature $T_{S}=5762 \mathrm{~K}$. The sun's rays reach the earth through a conical manifold, whose solid angle is a 46000th part of the celestial hemisphere. The modelled incident solar photon flux is therefore

$\Phi_{\text {inc }}(\hbar \omega)=C \frac{1}{46,000} \frac{2 \pi(\hbar \omega)^{2}}{h^{3} c^{2}} f_{B, 0 T s}(\hbar \omega) \frac{1000}{1360}$

The factor of $1000 / 1360$ is a correction to allow for absorption by the earth's atmosphere; this normalises the spectrum to a total irradiance of $1000 \mathrm{~W} \mathrm{~m}^{-2}$ as the usual standard at the Earth's surface (in the absence of concentration). $C$ is the concentration factor, which accounts for any concentrating optics focusing sunlight on the cell. Throughout this paper, we will take $C=1000 \times$ unless otherwise stated.

The input parameters used in the model are given in Table A1.

\section{Appendix B. adaptation of the model to allow for stronger absorbance of the QD layer stack}

In this appendix, all equations written (S-) refer to the supporting information of Ref. [34]. In the initial derivation of the net current between two levels, the exponential function in
Table A1

Input parameters used in detailed balance model.

\begin{tabular}{|c|c|c|}
\hline Symbol & Value & Units \\
\hline$E_{C B}$ & 0 & $\mathrm{eV}$ \\
\hline$E_{V B}$ at $300 \mathrm{~K}$ & -1.42 & $\mathrm{eV}$ \\
\hline$E_{V B, e f f}$ at $300 \mathrm{~K}$ & -1.24 & $\mathrm{eV}$ \\
\hline$E_{I B\left(\begin{array}{lll}1 & 1 & 1\end{array}\right)}$ & -0.26 & $\mathrm{eV}$ \\
\hline$\left.E_{I B(} \begin{array}{lll}2 & 1 & 1\end{array}\right)$ & -0.16 & $\mathrm{eV}$ \\
\hline 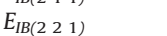 & -0.06 & $\mathrm{eV}$ \\
\hline$W$ & 2.4 & $\mu \mathrm{m}$ \\
\hline$n$ & 3.5 & \\
\hline$\rho_{Q D}$ & $5 \times 10^{15}$ & $\mathrm{~cm}^{-3}$ \\
\hline$N_{d}$ & $5 \times 10^{15}$ & $\mathrm{~cm}^{-3}$ \\
\hline$E_{d}$ & -0.006 & $\mathrm{eV}$ \\
\hline$N_{C B}$ & $8.4 \times 10^{13} T_{c}^{3 / 2}$ & $\mathrm{~cm}^{-3}$ \\
\hline$N_{V B}$ & $1.8 \times 10^{15} T_{c}^{3 / 2}$ & $\mathrm{~cm}^{-3}$ \\
\hline
\end{tabular}

The energy origin is at the host $\mathrm{CB}$ band edge. $T_{C}$ is expressed in degrees Kelvin.

Eq. (S9) was expanded in a Taylor series up to the first order. This is justified in the limit of low absorption. Here, the derivation is modified to allow for higher absorptions.

It will be convenient to make the following definitions:

$\beta_{\mathbf{k}, \varepsilon}=\alpha_{\mathbf{k}, \varepsilon}^{\max }\left(f_{l}-f_{u}\right) W$

$\beta_{x, y}=\alpha_{x, y}^{\max }\left(f_{l}-f_{u}\right) W$

$\beta_{z}=\alpha_{z}^{\max }\left(f_{l}-f_{u}\right) W$

where $J_{l \rightarrow u}^{L}$ and $J_{l \rightarrow u}^{D}$ are the illumination and dark currents that make up the total current for a given transition. The illumination current contains all terms proportional to the illuminating photon flux and the dark current contains all other terms.

Working through steps (S9) to (S13) of the supporting information in [34], without taking the Taylor expansion of the exponential, we have

$J_{l \rightarrow u}^{L}=q_{e} \int_{0}^{E_{g}} d E_{\text {phot }} \frac{c}{n} \rho_{\text {phot }} I^{L}$

$J_{l \rightarrow u}^{D}=q_{e} \int_{0}^{E_{g}} d E_{p h o t} \frac{c}{n} \rho_{p h o t}\left(f_{B, \mu T_{c}}-f_{B, 0 T_{c}}\right) I^{D}$

where $I^{L}$ and $I^{D}$ represent the following integrals

$I^{L}=\int_{0}^{2 \pi} \int_{0}^{\theta_{c}} d \phi d \theta \cos \theta \sin \theta\left(1-\exp \left[-\frac{\left\langle\beta_{\mathbf{k}, \varepsilon}\right\rangle_{\varepsilon}}{\cos \theta}\right]\right) N_{i n c}$

$I^{D}=\left[\begin{array}{l}\int_{0}^{2 \pi} \int_{0}^{\theta_{c}} d \phi d \theta \cos \theta \sin \theta\left(1-2 \exp \left[-\frac{\left\langle\beta_{\mathbf{k}, \varepsilon}\right\rangle_{\varepsilon}}{\cos \theta}\right]+\exp \left[-\frac{2\left\langle\beta_{\mathbf{k}, \varepsilon}\right\rangle_{\varepsilon}}{\cos \theta}\right]\right)+ \\ \int_{0}^{2 \pi} \int_{0}^{\pi / 2} d \phi d \theta \cos \theta \sin \theta\left(1-\exp \left[-\frac{2\left\langle\beta_{\mathbf{k}, \varepsilon}\right\rangle_{\varepsilon}}{\cos \theta}\right]\right)\end{array}\right]$

Returning to Eq. (S19), we observe that the QDs in the QD-IBSC under investigation have $x y$ symmetry, such that $\alpha_{x}^{\max }=\alpha_{y}^{\max }=$ $\alpha_{x, y}^{\max }$. Eq. (S19) then becomes

$$
\begin{aligned}
\left\langle\alpha_{\mathbf{k}, \varepsilon}^{\max }\right\rangle_{\varepsilon}= & \left(1-\frac{\sin ^{2} \theta_{k}}{2}\right) \alpha_{x, y}^{\max }+\frac{\sin ^{2} \theta_{k}}{2} \alpha_{z}^{\max } \\
& -\cos \phi_{k} \sin \phi_{k} \sin ^{2} \theta_{k} \alpha_{x, y}^{\max } \\
& -\left(\sin \phi_{k}+\cos \phi_{k}\right) \cos \theta_{k} \sin \theta_{k} \sqrt{\alpha_{z}^{\max } \alpha_{x, y}^{\max }}
\end{aligned}
$$

Clearly, the many sine and cosine terms in $\left\langle\alpha_{k, \varepsilon}^{\max }\right\rangle_{\varepsilon}$ make the integrals $I^{L}$ and $I^{D}$ very difficult to compute. For $I^{L}$, we can take a Taylor expansion of the bracketed expression up to an arbitrary order. We observe that all terms but one in the integrand are then proportional to $\sin \theta$ to the second power or higher. Making the approximation that these terms integrate to zero over the interval $0 \leq \theta \leq \theta_{c}$, the integral becomes

$I^{L}=\int_{0}^{2 \pi} \int_{0}^{\theta_{c}} d \phi d \theta \sin \theta\left(1-\exp \left[-\beta_{x, y}\right]\right) N_{i n c}$ 


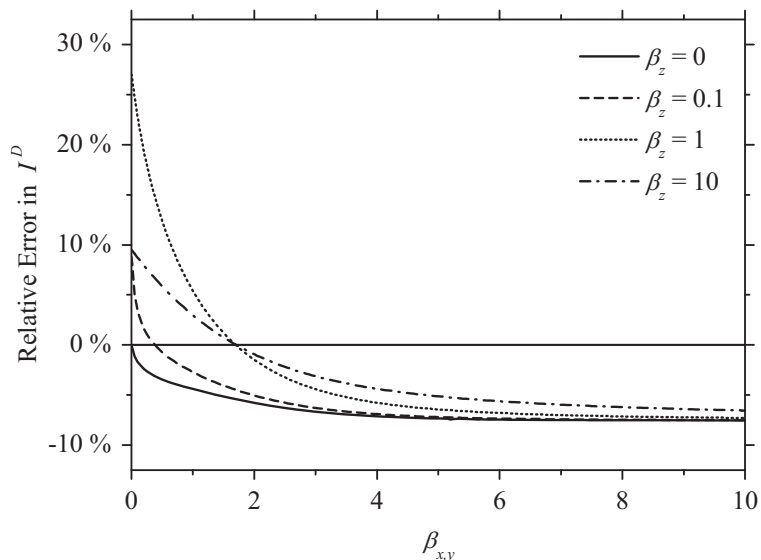

Fig. B1. Relative error induced by using approximate $I^{D}$ expressed in Eq. (B7) instead of the exact $I^{D}$ expressed in Eq. (B3), for a range of values of $\beta_{x, y}$ and $\beta_{z}$.

where we have also made the approximation $\cos \theta \approx 1$, which is reasonable throughout the interval of integration. The illumination current for the transition is therefore

$J_{l \rightarrow u}^{L}=q_{e} \int_{0}^{E_{g}} d E_{p h o t} \Phi_{i n c}\left(1-\exp \left[-\beta_{x, y}\right]\right)$

as we would expect. Eq. (B6) appears as part of Eq. (A2) in Appendix A with $\beta_{x, y}=\alpha_{x, y} W$, which can be verified from inspection of Eqs. (A3) and (B1).

For $I^{D}$ we can make a two approximations, both of which are justified numerically further on. First, we take the first integral in the expression for $I^{D}$ (Eq. (B3)) to be negligible compared to the second integral. Second, we perform the second integral taking $\left\langle\alpha_{k, \varepsilon}\right\rangle_{\varepsilon}$ to be simply the mean value of $\alpha_{x}^{\max }, \alpha_{y}^{\max }$ and $\alpha_{z}^{\max }$. Under these approximations, $I^{D}$ becomes

$$
\begin{aligned}
I^{D} & =\left[\int_{0}^{2 \pi} \int_{0}^{\pi / 2} d \phi d \theta \cos \theta \sin \theta\left(1-\exp \left[-\frac{2 \beta_{\text {iso }}}{\cos \theta}\right]\right)\right] \\
& =\pi\left(1-\left(1-2 \beta_{\text {iso }}\right) \exp \left[-2 \beta_{\text {iso }}\right]+4 \beta_{\text {iso }}^{2} E i\left[-2 \beta_{\text {iso }}\right]\right)
\end{aligned}
$$

where

$\beta_{\text {iso }}:=\frac{2 \beta_{x, y}+\beta_{z}}{3}$

and $\operatorname{Ei}[x]$ is the exponential integral defined by

$\operatorname{Ei}[x]=-\int_{-x}^{\infty} d t \frac{e^{-t}}{t}$

To justify the approximations made in the expression of $I^{D}$, Fig. B1 shows the relative difference between the approximate $I^{D}$ expressed in Eq. (B7) and the exact $I^{D}$ expressed in Eq. (B3), for a range of values of $\beta_{x, y}$ and $\beta_{z}$. The exact $I^{D}$ is calculated numerically. The relative error is in most cases below $10 \%$, but approaches almost $30 \%$ if the absorption is much stronger for photons propagating in the $z$ direction than in the $x y$ plane. In all cases, the error is tolerable for the semi-quantitative analysis performed here.

Computationally, it is far preferable to use the approximate $I^{D}$. The exact $I^{D}$ requires a numerical integral that would have to be executed in every iteration of the numerical solver used to find the QFLs. The approximate $I^{D}$ is not quite analytical, since it contains the exponential integral Ei $[x]$; however, software packages such as Mathematica or Matlab will calculate its value using a truncated series, which is much faster than performing a numerical integration.
The dark current can now be expressed as

$J_{l \rightarrow u}^{D}=q_{e} \int_{0}^{E_{g}} d E_{p h o t} \frac{2 \pi n^{2}\left(E_{\text {phot }}\right)^{2}}{h^{3} c^{2}}\left(f_{B, \mu T_{c}}-f_{B, 0 T_{c}}\right) I^{D}\left(\beta_{i s o}\right)$

Eq. (B10) appears as part of Eq. (A2) in Appendix A with $\beta_{i s o}=\alpha_{i s o} W$, which can be verified from inspection of Eqs. (A3), (B1) and (B8).

\section{References}

[1] A. Luque, A. Martí, Increasing the efficiency of ideal solar cells by photon induced transitions at intermediate levels, Phys. Rev. Lett. 78 (1997) 5014-5017.

[2] W. Shockley, H.J. Queisser, Detailed balance limit of efficiency of p-n junction solar cells, J. Appl. Phys. 32 (1961) 510-519.

[3] A. Martí, L. Cuadra, A. Luque, Quantum dot intermediate band solar cell, in: 28th IEEE Photovoltaic Specialists Conference, Anchorage (Alaska), 2000, pp. 940-943.

[4] A. Luque, A. Marti, C. Stanley, N. Lopez, L. Cuadra, D. Zhou, J.L. Pearson, A. McKee, General equivalent circuit for intermediate band devices: potentials, currents and electroluminescence, J. Appl. Phys. 96 (2004) 903-909.

[5] S.M. Hubbard, C.D. Cress, C.G. Bailey, R.P. Raffaelle, S.G. Bailey, D.M. Wilt, Effect of strain compensation on quantum dot enhanced GaAs solar cells, Appl. Phys. Lett. 92 (2008) 123512-123513.

[6] S. Blokhin, A. Sakharov, A. Nadtochy, A. Pauysov, M. Maximov, N. Ledentsov, A. Kovsh, S. Mikhrin, V. Lantratov, S. Mintairov, N. Kaluzhniy, M. Shvarts, AlGaAs/GaAs photovoltaic cells with an array of InGaAs QDs, Semiconductors 43 (2009) 514-518.

[7] V. Popescu, G. Bester, M.C. Hanna, A.G. Norman, A. Zunger, Theoretical and experimental examination of the intermediate-band concept for strainbalanced (In,Ga)As/Ga(As,P) quantum dot solar cells, Phys. Rev. B: Condens. Matter 78 (2008) 205321.

[8] R. Oshima, A. Takata, Y. Okada, Strain-compensated InAs/GaNAs quantum dots for use in high-efficiency solar cells, Appl. Phys. Lett. 93 (2008) 083111-083113.

[9] D. Alonso-Alvarez, A.G. Taboada, J.M. Ripalda, B. Alen, Y. Gonzalez, L. Gonzalez, J.M. Garcia, F. Briones, A. Marti, A. Luque, A.M. Sanchez, S.I. Molina, Carrier recombination effects in strain compensated quantum dot stacks embedded in solar cells, Appl. Phys. Lett. 93 (2008) 123114.

[10] W. Shan, W. Walukiewicz, J.W. Ager, E.E. Haller, J.F. Geisz, D.J. Friedman, J.M. Olson, S.R. Kurtz, Band anticrossing in GaInNAs alloys, Phys. Rev. Lett. 82 (1999) 1221-1224.

[11] N. Ahsan, N. Miyashita, M. Monirul Islam, K. Man, Yu, W. Walukiewicz, Y. Okada, Two-photon excitation in an intermediate band solar cell structure, Appl. Phys. Lett. 100 (2012) 172111.

[12] T. Tanaka, M. Miyabara, Y. Nagao, K. Saito, G. Qixin, M. Nishio, Y. Kin Man, W. Walukiewicz, Photogenerated current by two-step photon excitation in ZnTeO intermediate band solar cells with $n$-ZnO window layer, IEEE J. Photovoltaics 4 (2014) 196-201.

[13] A. Luque, A. Martí, E. Antolín, C. Tablero, Intermediate bands versus levels in non-radiative recombination, Physica B 382 (2006) 320-327.

[14] B. Marsen, S. Klemz, T. Unold, H.-W. Schock, Investigation of the sub-bandgap photoresponse in CuGaS2:Fe for intermediate band solar cells, Prog. Photovoltaics Res. Appl. 20 (2012) 625-629.

[15] P.G. Linares, A. Martí, E. Antolín, I. Ramiro, E. López, E. Hernández, D. Fuertes Marrón, I. Artacho, I. Tobías, P. Gérard, C. Chaix, R.P. Campion, C.T. Foxon, C.R. Stanley, S.I. Molina, A. Luque, Extreme voltage recovery in GaAs:Ti intermediate band solar cells, Sol. Energy Mater. Sol. Cells 108 (2013) 175-179.

[16] A. Luque, A. Marti, L. Cuadra, Thermodynamic consistency of sub-bandgap absorbing solar cell proposals, IEEE Trans. Electron Devices 48 (2001) 2118-2124.

[17] L. Cuadra, A. Marti, A. Luque, Influence of the overlap between the absorption coefficients on the efficiency of the intermediate band solar cell, IEEE Trans. Electron Devices 51 (2004) 1002-1007.

[18] L. Cuadra, A. Martí, N. López, A. Luque, Phonon bottleneck effect and photon absorption in self-ordered quantum dot intermediate band solar cells, in: 19th European Photovoltaic Solar Energy Conference and Exhibition, Paris, 2004, pp. 250-253.

[19] A. Martí, E. Antolín, C.R. Stanley, C.D. Farmer, N. López, P. Díaz, E. Cánovas, P.G. Linares, A. Luque, Production of Photocurrent due to Intermediate-toConduction-Band Transitions: a Demonstration of a Key Operating Principle of the Intermediate-Band Solar Cell, Phys. Rev. Lett. 97 (2006) 247701.

[20] P.G. Linares, A. Martí, E. Antolín, I. Ramiro, A. Luque, Voltage recovery in intermediate band solar cells, Sol. Energy Mater. Sol. Cells 98 (2011) 240-244.

[21] I. Ramiro, A. Martí, A. Luque, E. Antolín, Review of Experimental Results Related to the Operation of Intermediate Band Solar Cells, IEEE J. Photovoltaics (2014).

[22] E. Antolín, A. Marti, C.D. Farmer, P.G. Linares, E. Hernandez, A.M. Sanchez T. Ben, S.I. Molina, C.R. Stanley, A. Luque, Reducing carrier escape in the InAs/ GaAs quantum dot intermediate band solar cell, J. Appl. Phys. 108 (2010) 064513-064517.

[23] I. Ramiro, E. Antolin, M.J. Steer, P.G. Linares, E. Hernandez, I. Artacho, E. Lopez, T. Ben, J.M. Ripalda, S.I. Molina, F. Briones, C.R. Stanley, A. Marti, A. Luque, InAs/AlGaAs quantum dot intermediate band solar cells with enlarged 
sub-bandgaps, in: Photovoltaic Specialists Conference (PVSC), 2012 38th IEEE, 2012, pp. 000652-000656.

[24] C.G. Bailey, D.V. Forbes, S.J. Polly, Z.S. Bittner, Y. Dai, C. Mackos, R.P. Raffaelle, S. M. Hubbard, Open-circuit voltage improvement of InAs/GaAs quantum-dot solar cells using reduced InAs coverage, IEEE J. Photovoltaics 2 (2012) 269-275.

[25] A. Takata, R. Oshima, Y. Shoji, K. Akahane, Y. Okada, Fabrication of 100 layerstacked InAs/GaNAs strain-compensated quantum dots on GaAs (001) for application to intermediate band solar cell, in: Photovoltaic Specialists Conference (PVSC), 2010 35th IEEE, 2010, pp. 001877-001880.

[26] K. Akahane, N. Yamamoto, T. Kawanishi, Fabrication of ultra-high-density InAs quantum dots using the strain-compensation technique, Phys. Status Solidi A 208 (2011) 425-428.

[27] I. Tobias, C. del Cañizo, J. Alonso, Crystalline silicon solar cells and modules, in: A. Luque, S. Hegedus (Eds.), Handbook of Photovoltaic Science and Engineering, John Wiley \& Sons, London, 2003, pp. 255-306.

[28] M.A. Green, Silicon solar-cells-evolution, high-efficiency design and efficiency enhancements, Semicond. Sci. Technol. 8 (1993) 1-12.

[29] B. Sopori, Thin-film silicon solar cells, in: A. Luque, S. Hegedus (Eds.), Handbook of Photovoltaic Science and Engineering, John Wiley \& Sons, Chichester 2003, pp. 307-354.

[30] S. Tomic, T.S. Jones, N.M. Harrison, Absorption characteristics of a quantum dot array induced intermediate band: implications for solar cell design, Appl. Phys. Lett. 93 (2008) (263105-263103).

[31] A. Luque, A. Marti, E. Antolin, P. Garcia-Linares, Intraband absorption for normal illumination in quantum dot intermediate band solar cells, Sol. Energy Mater. Sol. Cells 94 (2010) 2032-2035.

[32] A. Luque, A. Martí, E. Antolín, P.G. Linares, I. Tobías, I. Ramiro, E. Hernandez New Hamiltonian for a better understanding of the quantum dot intermediate band solar cells, Sol. Energy Mater. Sol. Cells 95 (2011) 2095-2101.

[33] A. Luque, A. Marti, E. Antolin, P.G. Linares, I. Tobias, I. Ramiro, Radiative thermal escape in intermediate band solar cells, AIP Adv. 1 (2011) 022125-022126.

[34] A. Mellor, A. Luque, I. Tobías, A. Martí, Realistic detailed balance study of the quantum efficiency of quantum dot solar cells, Adv. Funct. Mater. 24 (2014) 339-345.

[35] E. Antolín, A. Marti, P.G. Linares, I. Ramiro, E. Hernandez, C.D. Farmer, C.R. Stanley, A. Luque, Advances in quantum dot intermediate band solar cells, in: 35th IEEE Photovoltaic Specialists Conference (PVSC), Honolulu, HI, USA, 2010, pp. 000065-000070.

[36] P. Linares, Research on Intermediate Band Solar Cells and Development of Experimental Techniques for their Characterization under Concentrated Illumination, Universidad Politecnica de Madrid, 2012.

[37] A. Luque, E. Antolín, P.G. Linares, I. Ramiro, A. Mellor, I. Tobías, A. Martí, Interband optical absorption in quantum well solar cells, Sol. Energy Mater. Sol. Cells 112 (2013) 20-26.
[38] D. Zhou, G. Sharma, S.F. Thomassen, T.W. Reenaas, B. Fimland, Optimization towards high density quantum dots for intermediate band solar cells grown by molecular beam epitaxy, Appl. Phys. Lett. 96 (2010) (061913-061913).

[39] H. Fujita, K. Yamamoto, J. Ohta, Y. Eguchi, K. Yamaguchi, In-plane quantum-dot superlattices of InAs on GaAsSb/GaAs(001) for intermediate band solar-cells, in: Photovoltaic Specialists Conference (PVSC), 2011 37th IEEE, 2011, pp. 002612-002614.

[40] L. Zeng, P. Bermel, Y. Yi, B.A. Alamariu, K.A. Broderick, J. Liu, C. Hong, X. Duan, J. Joannopoulos, L.C. Kimerling, Demonstration of enhanced absorption in thin film Si solar cells with textured photonic crystal back reflector, Appl. Phys. Lett. 93 (2008) (221105-221103).

[41] O. Berger, D. Inns, A.G. Aberle, Commercial white paint as back surface reflector for thin-film solar cells, Sol. Energy Mater. Sol. Cells 91 (2007) $1215-1221$.

[42] G. Jin, P.I. Widenborg, P. Campbell, S. Varlamov, Lambertian matched absorption enhancement in PECVD poly-Si thin film on aluminum induced textured glass superstrates for solar cell applications, Prog. Photovoltaics Res. Appl. 18 (2010) 582-589.

[43] M.A. Green, Two new efficient crystalline silicon light-trapping textures, Prog. Photovoltaics Res. Appl. 7 (1999) 317-320.

[44] C. Heine, R.H. Morf, Submicrometer gratings for solar energy applications, Appl. Opt. 34 (1995) 2476-2482.

[45] H.A. Atwater, A. Polman, Plasmonics for improved photovoltaic devices, Nat. Mater. 9 (2010) 205-213.

[46] J. Nelson, The Physics of Solar Cells, Imperial College Press, 2003.

[47] A. Mellor, I. Tobías, A. Martí, M.J. Mendes, A. Luque, Upper limits to absorption enhancement in thick solar cells using diffraction gratings, Prog. Photovoltaics Res. Appl. 19 (2011) 676-687.

[48] C. Ulbrich, S. Fahr, J. Üpping, M. Peters, T. Kirchartz, C. Rockstuhl, R. Wehrspohn, A. Gombert, F. Lederer, U. Rau, Directional selectivity and ultra-light-trapping in solar cells, Phys. Status Solidi A 205 (2008) 2831-2843.

[49] Z. Yu, A. Raman, S. Fan, Fundamental limit of nanophotonic light trapping in solar cells, Proc. Nat. Acad. Sci. U.S.A. 107 (2010) 17491-17496.

[50] A. Luque, S. Hegedus, Handbook of Photovoltaic Science and Engineering, John Wiley \& Sons, Chichester, 2003.

[51] R. Winston, W.T. Welford, Optics of Non Imaging Concentrators, Academic, 1979.

[52] A. Luque, A. Martí, A. Mellor, D. Fuertes Marrón, I. Tobías, E. Antolín, Absorption coefficient for the intraband transitions in quantum dot materials, Prog. Photovoltaics Res. Appl. 21 (2013) 658-667.

[53] A. Luque, A. Mellor, E. Antolín, P.G. Linares, I. Ramiro, I. Tobías, A. Martí, Symmetry considerations in the empirical k.p Hamiltonian for the study of intermediate band solar cells, Sol. Energy Mater. Sol. Cells 103 (2012) 171-183.

[54] A. Luque, A. Mellor, I. Ramiro, E. Antolín, I. Tobías, A. Martí, Interband absorption of photons by extended states in intermediate band solar cells, Sol. Energy Mater. Sol. Cells 115 (2013) 138-144.

[55] A. Luque, A. Marti, On the partial filling of the intermediate band in IB solar cells, IEEE Trans. Electron Devices 57 (2010) 1201-1207. 